\title{
PARIS, LYONS AND MEDITERRANEAN
} RAILWAY.

(Chemins de fer de Paris à Lyon et à la Méditerranée.)

Mr. L. MARÉCHAL.

Mr. L. Maréchal, Locomotive and Rolling Stock Superintendent, wrote that he could not for the present make any definite statement regarding warning and signal-recording apparatus on the line, as 
these had but recently been fixed on a few engines running on certain sections and were being experimented upon.

Mr. Maréchal referred besides to the difficulties inherent to the winter season, and suggested that it would be premature at this stage to say how eflicient the apparatus was likely to be. This male him desirous not to make any statement before a long trial had taken place. But he would add that experiments were being carried out on 140 express locomotives and on 415 kilometres (258 miles) of double track with 316 disks fully equipped.

Though it was not possible to describe the apparatus, owing to the continual improvements carried out and the constant changes, yet he was willing to give the general principle on which it worked.

\section{[Translated.]}

The warning and recording apparatus for signals at "danger," which is now being experimented upon on the Paris, Lyons and Mediterranean Railway works thus when the engine goes over a ramp :-

1. It actuates a special whistle which warns the driver and the stoker.

2. It traces on the roll of the speed-indicator a conventional sign, the position of which fixes the signal which was at danger. Both these results are obtained simultaneously by means of an assemblage of devices placed on the track and on the engine.

The apparatus placed on the track comprises an insulated conductor, called a "crocodile" or ramp, fixed between the rails near the signal corresponding to it, a switch worked by the same signal and connected to the ramp and the rails.

The apparatus on the engine consists of :-

A brush which rubs on the ramp when the engine passes over it.

A pneumatic horn giving the warning.

An automatic device for unclutching and sending compressed air through the horn.

An automatic device acting on the registering mechanism of the speed-indicator. 
An electro-pneumatic relay which provides the unclutching device at the horn and the registering apparatus with the compressed air required.

A battery which provides the electro-pneumatic relay with the current required.

A compressed-air receiver which feeds the apparatus. (On engines already provided with pneumatic brakes no extra receiver is necessary - the one used for the brakes serving both purposes.)

This warning and registering system is based on the combined use of electricity and compressed air. It makes it necessary to use switches and ramps similar to those adopted on other railways where the system is entirely electrical. In this latter case, electricity is the medium which brings about the unclutching of the apparatus during the very short time that the engine takes to pass over a ramp. This unclutching means a certain force being exercised, which, however weak, is not a negligible quantity, so that at very high speeds there might easily be failures.

In the Paris, Lyons and Mediterranean system, electricity comes into play, only to release the armature of the electro-magnet of the relay and close a circuit on the engine itself, without doing any mechanical work. Through this local circuit of the electropneumatic relay (which is fed electrically from the battery on the engine and pneumatically from the receiver) being closed, the compressed air becomes the agent which definitely provides the mechanical power required for unclutching and reclutching. 